\title{
DYNAMIC AND RESONANCE RESPONSE ANALYSIS FOR A TURBINE BLADE WITH VARYING ROTATING SPEED
}

\author{
Dan Wang, Zhifeng HaO \\ School of Mathematical Sciences, University of Jinan, Jinan 250022, China \\ e-mail: danwang2014518@hotmail.com \\ YUSHU CHEN \\ School of Astronautics, Harbin Institute of Technology, Harbin 150001, China
}

YONGXIANG ZHANG

School of Mathematical Sciences, University of Jinan, Jinan 250022, China

\begin{abstract}
A coupling model between turbine blades with a varying rotating speed and oncoming vortices is constructed, where the coupling of the structure and the fluid is simulated by the van der Pol oscillation. Partial differential governing equations of motions for the coupled system are obtained and discretized by using the Galerkin method. The 1:2 subharmonic resonance and the 1:1 internal resonance are investigated with the multiple scale method and first-order averaged equations are then derived. Nonlinear responses and bifurcation characteristics are studied by a numerical integration method. Stability of bifurcation curves is determined by utilizing the Routh-Hurwitz criterion. The effect of system parameters including the detuning parameter, steady-state rotating speed, amplitude of periodic perturbation for the rotating speed and freestream velocity on vibration responses are investigated.
\end{abstract}

Keywords: varying rotating speed, van der Pol oscillation, multiple scale method, nonlinear response, bifurcation curve

\section{Introduction}

The blade is an important component in the turbomachinery, such as gas axial compressors, wind turbines, aero-engine turbines, etc. Rotating blades are subjected to high centrifugal and aerodynamic loads which can lead to aeroelastic problems of the blades, like flutter and vortex-induced vibrations (Gostelow et al., 2006). To keep safe running of the turbomachinery, analysis of the dynamic response characteristics is of importance for the blade design.

Owing to a variety of engineering applications, dynamic analysis of rotating blades has received broad interest. Transverse and rotational motion as well as control of vibrations for a rotating uniform Euler-Bernoulli beam were studied by Yang et al. (2004). In addition, the early research that focused on the problem of rotating nonconstant speed was done in the work by Kammer and Schlack (1987). Nonlinear vibration of a variable speed rotating beam was studied by Younesian and Esmailzadeh (2010), where the influence of various parameters was investigated. The nonlinear dynamic response of a rotating blade with varying rotating speed was investigated by Yao et al. (2012), and the results showed that the dynamic responses of the rotating blade changed from periodic motions to chaotic motions with different rotating speeds. Nonlinear oscillations and steady-state responses of a rotating compressor blade with varying rotating speed were investigated by Yao et al. (2014). Staino and Basu (2013) formulated a multi-modal flexible wind turbine model with variable rotor speed by using a Lagrangian approach, and anlysed the effect of the rotational speed on the edgewise vibration of the blades. The equations of motion of a rotating composite Timoshenko beam were derived in the study of 
Georgiades et al. (2014), and the results showed that the variable rotating speed as well as a nonzero pitch angle have important effects on the system dynamics. A more accurate nonlinear model of a rotating cantilever beam was proposed by Kim and Chung (2016). Geometrically nonlinear vibrations of beams with properties periodically varying along the axis were investigated by Domagalski and Jędrysiak (2016). A new model for a spinning beam under deployment was proposed and the dynamic responses and characteristics were analyzed by Zhu and Chung (2016). The study of forced nonlinear vibrations of a simply supported Euler-Bernoulli beam resting on a nonlinear elastic foundation with quadratic and cubic nonlinearities was carried out by Shahlaei-Far et al. (2016) with the homotopy analysis method. Vibration of a rotating beam with variable speed/acceleration has been controlled by using the sandwich beam filled with an ER fluid (Wei et al., 2006). Moreover, Warmiński and Latalski (2016) applied a nonlinear saturation control strategy to suppress vibration of the rotating hub-beam structure.

The vortex-induced vibration of a rotating blade with the steady-state rotating speed was investigated by Wang et al. (2016c), where the time-varying characteristic of the vortex shedding was represented by a van der Pol oscillator. Moreover, the van der Pol oscillator has been introduced as a reduced model in a number of articles to model the time-varying characteristics of the fluid (Hartlen and Currie, 1970; Barron and Sen, 2009; Hemon, 1999; Gabbai and Benaroya, 2005; Wang et al., 2016a) or the fluid-structure interaction (Barron, 2010; Lee et al., 2006; Facchinetti et al., 2004; Keber and Wiercigroch, 2008; Wang et al., 2016b) according to experimental and numerical studies. In addition, the effect of structural vibration on the motion of the fluid was also investigated in the above articles. Under different air flow conditions, the dynamic behaviour of the blades becomes very complex when the rotating speed is time-varying, which could convert to a nonlinear system with the coupling of parametric-excitation and self-excitation.

The motivation of this paper is to investigate the dynamic response and bifurcation characteristics of blades with varying rotating speed. The coupling model of the blade with the varying rotating speed and the time-varying flows is derived based on the results by Wang et al. (2016c). The analysis of the 1:2 subharmonic resonance and 1:1 internal resonance is carried out with the multiple scale method. Four-dimensional nonlinear averaged equations are then derived. Bifurcation curves are obtained and the effect of the system parameters on dynamic responses are discussed in detail.

\section{Modeling}

\subsection{Modeling of the coupling for the structure and vortices}

The blade with length $r$ and varying rotating speed $\Omega$ is assumed as a continuous uniform straight cantilever beam based on the Euler-Bernoulli formulation in the centrifugal force field as shown in Fig. 1.

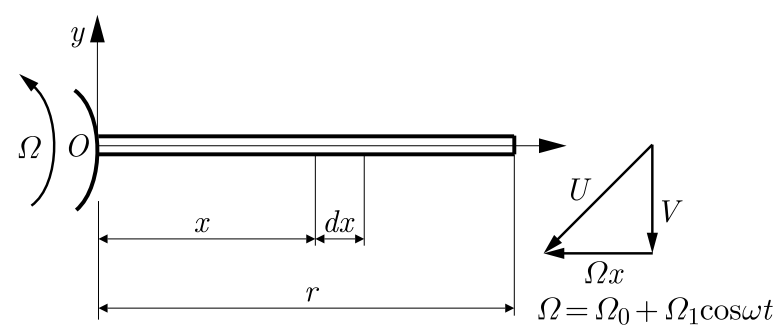

Fig. 1. A beam with varying rotating speed

Similar to the derivation process of formulas in the study by Wang et al. (2016c), the governing equation of transverse motion of a uniform cantilever beam with varying rotating speed can be obtained as follows 


$$
\begin{aligned}
& E I \frac{\partial^{4} w(x, t)}{\partial x^{4}}+\widetilde{m} \frac{\partial^{2} w(x, t)}{\partial t^{2}}+\widetilde{c} \frac{\partial w(x, t)}{\partial t} \\
& \quad=F_{f}-\rho A \Omega^{2} x \frac{\partial w(x, t)}{\partial x}+\frac{1}{2} \rho A \Omega^{2}\left(r^{2}-x^{2}\right) \frac{\partial^{2} w(x, t)}{\partial x^{2}}
\end{aligned}
$$

where $w(x, t)$ denotes the transverse displacement of the blade, $E I$ is the flexural rigidity of the structure, $\widetilde{c}$ is the viscous damping coefficient, $\widetilde{m}=\left(\rho+\rho_{f}\right) A$ is the total mass of the structure and fluid, $\rho$ and $\rho_{f}$ are densities of the structure and air flow, respectively, $A$ is the area of the cross-section of the cantilever beam, $F_{f}=0.5 \rho_{f} U^{2} \bar{D} C_{L}(x, t)$ is the lift force effecting the blade and induced by the vortex, $U=\sqrt{V^{2}+(\Omega x)^{2}}$ is the total velocity, $V$ is the freestream velocity, $\bar{D}$ denotes characteristic length of the cross-section of the beam. Here, the varying rotating speed is expressed as $\Omega=\Omega_{0}+\Omega_{1} \cos \omega t$, representing the periodic perturbation $\Omega_{1} \cos \omega t$ on the steady-state rotating speed $\Omega_{0}$.

Letting $v(x, t)=w(x, t) / \bar{D}, z=x / r$, Eq. (2.1) can be rewritten as

$$
\begin{aligned}
& \omega_{0}^{2} \frac{\partial^{4} v(z, t)}{\partial z^{4}}+\frac{\partial^{2} v(z, t)}{\partial t^{2}}+\zeta \frac{\partial v(z, t)}{\partial t} \\
& \quad=\frac{1}{4 \widetilde{m}} C_{L 0} \rho_{f}\left[V^{2}+(r \Omega z)^{2}\right] q(z, t)-\frac{\rho A \Omega^{2}}{\widetilde{m}} z \frac{\partial v(z, t)}{\partial z}+\frac{\rho A \Omega^{2}}{2 \widetilde{m}}\left(1-z^{2}\right) \frac{\partial^{2} v(z, t)}{\partial z^{2}}
\end{aligned}
$$

with the boundary conditions $v(0, t)=0, v^{\prime}(0, t)=0, v^{\prime \prime}(1, t)=0, v^{\prime \prime \prime}(1, t)=0$, where $\omega_{0}=\sqrt{E I /\left(\widetilde{m} r^{4}\right)}, \zeta=\widetilde{c} / \widetilde{m}$ is the damping ratio, $q(z, t)=2 C_{L} / C_{L 0}$ represents a time-varying variable of the vortical flows, $C_{L 0}$ is the reference lift coefficient.

Similarly, the van der Pol oscillator is applied to simulate time-varying characteristics of the vortices as follows

$$
\frac{\partial^{2} q(z, t)}{\partial t^{2}}+s \omega_{f}\left[q^{2}(z, t)-1\right] \frac{\partial q(z, t)}{\partial t}+\omega_{f}^{2} q(z, t)=M \frac{\partial^{2} v(z, t)}{\partial t^{2}}
$$

where $\omega_{f}$ is the shedding frequency of the vortex, $s$ is the van der Pol damping coefficient, $M$ is the linear coupling parameter representing the impact of structural vibration on the fluid motion.

\subsection{The Galerkin discretization of the coupled system}

Discretization of partial differential equations (2.2) and (2.3) into a finite-dimensional system is done according to the study by Clough and Penzien (2003), Wang et al. (2016c), letting

$$
v(z, t)=\sum_{i=1}^{\infty} v_{i}(t) \widetilde{v}_{i}(z)
$$

represent an arbitrary oscillation of the structure and

$$
q(z, t)=\sum_{i=1}^{\infty} q_{i}(t) \widetilde{q}_{i}(z)
$$

denote an arbitrary oscillation of the vortical flows.

The modal functions of the structure and the fluid are expressed as those used in the study of Wang et al. (2016c), that is

$$
\widetilde{v}_{i}(z)=\cosh \left(\beta_{i} z\right)-\cos \left(\beta_{i} z\right)-\frac{\cosh \beta_{i}+\cos \beta_{i}}{\sinh \beta_{i}+\sin \beta_{i}}\left[\sinh \left(\beta_{i} z\right)-\sin \left(\beta_{i} z\right)\right]
$$

and

$$
\widetilde{q}_{i}(z)=\sin (i \pi z) \quad i=1,2, \ldots
$$


where $\beta_{i}(i=1,2, \ldots)$ satisfy the equation $\cos \beta \cosh \beta+1=0$ that is obtained from the boundary conditions for the cantilever beam.

Repeating the discretization process again, the first mode motion of the structure and fluid can be derived as follows

$$
\begin{aligned}
& \frac{d^{2} v_{1}(t)}{d t^{2}}+\zeta \frac{d v_{1}(t)}{d t}+\omega_{0}^{2} \beta_{1}^{4} v_{1}(t)+a\left(\Omega_{0}+\Omega_{1} \cos \omega t\right)^{2} v_{1}(t) \\
& \quad=\left[b+\widetilde{d}\left(\Omega_{0}+\Omega_{1} \cos \omega t\right)^{2}\right] q_{1}(t) \\
& \frac{d^{2} q_{1}(t)}{d t^{2}}+s \omega_{f}\left[\frac{3}{4} q_{1}^{2}(t)-1\right] \frac{d q_{1}(t)}{d t}+\omega_{f}^{2} q_{1}(t)=M \frac{d^{2} v_{1}(t)}{d t^{2}}
\end{aligned}
$$

where

$$
\begin{aligned}
& a=\frac{\rho A}{2 \widetilde{m}} \frac{2 \int_{0}^{1} \frac{\widetilde{v}_{1}(z)}{d z} \widetilde{v}_{1}(z) z d z-\int_{0}^{1} \frac{d^{2} \widetilde{v}_{1}(z)}{d z^{2}}\left(1-z^{2}\right) \widetilde{v}_{1}(z) d z}{\int_{0}^{1} \widetilde{v}_{1}^{2}(z) d z} \\
& b=\frac{C_{L 0} \rho_{f} V^{2}}{4 \widetilde{m}} \frac{\int_{0}^{1} \widetilde{q}_{1}(z) \widetilde{v}_{1}(z) d z}{\int_{0}^{1} \widetilde{v}_{1}^{2}(z) d z} \quad \widetilde{d}=\frac{C_{L 0} \rho_{f} r^{2}}{4 \widetilde{m}} \frac{\int_{0}^{1} \widetilde{q}_{1}(z) \widetilde{v}_{1}(z) z^{2} d z}{\int_{0}^{1} \widetilde{v}_{1}^{2}(z) d z}
\end{aligned}
$$

Equations (2.8) model the interactions between the vortical flows and the structure, which is also a Mathieu-van der Pol type oscillation.

\section{Analysis with the multiple scale method}

The research by Hao and Cao (2015), Hao et al. (2016) showed that nonlinear systems can present rich dynamic characteristics when the resonance occurs, like the primary resonance, superharmonic/subharmonic resonance as well as the internal resonance, etc. The multiple scale method is often utilized to understand qualitative characteristics of the system which present resonant conditions (Nayfeh and Mook, 1979).

Introducing the scaling parameters $\zeta \rightarrow \varepsilon \zeta, C_{L 0} \rightarrow \varepsilon C_{L 0}, s \rightarrow \varepsilon s, \Omega_{1} \rightarrow \varepsilon \Omega_{1}, M \rightarrow \varepsilon M$ into Eqs. (2.8), one can obtain

$$
\begin{aligned}
& \frac{d^{2} v_{1}(t)}{d t^{2}}+\varepsilon \zeta \frac{d v_{1}(t)}{d t}+\omega_{s}^{2} v_{1}(t)+a\left(\varepsilon^{2} \Omega_{1}^{2} \cos ^{2} \omega t+2 \varepsilon \Omega_{0} \Omega_{1} \cos \omega t\right) v_{1}(t) \\
& \quad=\left[\varepsilon b+\varepsilon \widetilde{d}\left(\Omega_{0}+\varepsilon \Omega_{1} \cos \omega t\right)^{2}\right] q_{1}(t) \\
& \frac{d^{2} q_{1}(t)}{d t^{2}}+\varepsilon s \omega_{f}\left[\frac{3}{4} q_{1}^{2}(t)-1\right] \frac{d q_{1}(t)}{d t}+\omega_{f}^{2} q_{1}(t)=\varepsilon M \frac{d^{2} v_{1}(t)}{d t^{2}}
\end{aligned}
$$

where $\omega_{s}=\sqrt{\omega_{0}^{2} \beta_{1}^{4}+a \Omega_{0}^{2}}$ denotes the uncoupled natural frequency of the first-order mode of the beam.

Considering the possible 1:1 internal resonance between the structure and the fluid as well as the 1:2 subharmonic resonance conditions, the relations of frequencies can be expressed as $\omega=2 \omega_{s}+\varepsilon \sigma, \omega_{f}=\omega_{s}+\varepsilon \sigma_{1}$, where $\sigma, \sigma_{1}$ are the detuning parameters, respectively.

Assume the approximate form of the solutions as shown in the following

$$
\begin{aligned}
& v_{1}(t)=v_{10}\left(T_{0}, T_{1}\right)+\varepsilon v_{11}\left(T_{0}, T_{1}\right)+\ldots \\
& q_{1}(t)=q_{10}\left(T_{0}, T_{1}\right)+\varepsilon q_{11}\left(T_{0}, T_{1}\right)+\ldots
\end{aligned}
$$

Substituting solutions (3.2) into Eqs. (3.1) and equating the coefficients of like powers of $\varepsilon$, one can obtain: 
— order $\varepsilon^{0}$

$$
\begin{aligned}
& D_{0}^{2} v_{10}\left(T_{0}, T_{1}\right)+\omega_{s}^{2} v_{10}\left(T_{0}, T_{1}\right)=0 \\
& D_{0}^{2} q_{10}\left(T_{0}, T_{1}\right)+\omega_{f}^{2} q_{10}\left(T_{0}, T_{1}\right)=0
\end{aligned}
$$

— order $\varepsilon^{1}$

$$
\begin{aligned}
& D_{0}^{2} v_{11}\left(T_{0}, T_{1}\right)+2 D_{0} D_{1} v_{10}\left(T_{0}, T_{1}\right)+\omega_{s}^{2} v_{11}\left(T_{0}, T_{1}\right)=b q_{10}\left(T_{0}, T_{1}\right)+\widetilde{d} \Omega_{0}^{2} q_{10}\left(T_{0}, T_{1}\right) \\
& \quad-\zeta D_{0} v_{10}\left(T_{0}, T_{1}\right)-2 a v_{10}\left(T_{0}, T_{1}\right) \Omega_{0} \Omega_{1} \cos \omega t \\
& D_{0}^{2} q_{11}\left(T_{0}, T_{1}\right)+2 D_{0} D_{1} q_{10}\left(T_{0}, T_{1}\right)+\omega_{f}^{2} q_{11}\left(T_{0}, T_{1}\right)=M D_{0}^{2} v_{10}\left(T_{0}, T_{1}\right) \\
& \quad-s \omega_{f}\left[\frac{3}{4} q_{10}^{2}\left(T_{0}, T_{1}\right)-1\right] D_{0} q_{10}\left(T_{0}, T_{1}\right)
\end{aligned}
$$

where

$$
\frac{d}{d t}=D_{0}+\varepsilon D_{1}+\varepsilon^{2} D_{2}+\ldots \quad \frac{d^{2}}{d t^{2}}=D_{0}^{2}+2 \varepsilon D_{0} D_{1}+\ldots \quad D_{n}=\frac{\partial}{\partial T_{n}}
$$

General solutions to Eqs. (3.3) can be obtained in the complex form

$$
\begin{aligned}
& v_{10}=A\left(T_{1}\right) \mathrm{e}^{\mathrm{i} \omega_{s} T_{0}}+\bar{A}\left(T_{1}\right) \mathrm{e}^{-\mathrm{i} \omega_{s} T_{0}} \\
& q_{10}=B\left(T_{1}\right) \mathrm{e}^{\mathrm{i} \omega_{f} T_{0}}+\bar{B}\left(T_{1}\right) \mathrm{e}^{-\mathrm{i} \omega_{f} T_{0}}
\end{aligned}
$$

Substituting (3.5) into Eqs. (3.4) and considering the resonance conditions yields

$$
\begin{gathered}
D_{0}^{2} v_{11}+\omega_{s}^{2} v_{11}=b B \mathrm{e}^{\mathrm{i}\left(\omega_{s} T_{0}+\sigma_{1} T_{1}\right)}+\widetilde{d} \Omega_{0}^{2} B \mathrm{e}^{\mathrm{i}\left(\omega_{s} T_{0}+\sigma_{1} T_{1}\right)}-\mathrm{i} \zeta \omega_{s} A \mathrm{e}^{\mathrm{i} \omega_{s} T_{0}} \\
-a \Omega_{0} \Omega_{1}\left[A \mathrm{e}^{\mathrm{i}\left(\omega+\omega_{s}\right) T_{0}}+\bar{A} \mathrm{e}^{\mathrm{i}\left(\omega_{s} T_{0}+\sigma T_{1}\right)}\right]-2 \mathrm{i} \omega_{s} D_{1} A \mathrm{e}^{\mathrm{i} \omega_{s} T_{0}}+c . c . \\
D_{0}^{2} q_{11}+\omega_{f}^{2} q_{11}=-M \omega_{s}^{2} A \mathrm{e}^{\mathrm{i}\left(\omega_{f}-\varepsilon \sigma_{1}\right) T_{0}}-2 \mathrm{i} \omega_{f} D_{1} B \mathrm{e}^{\mathrm{i} \omega_{f} T_{0}} \\
\quad-\mathrm{i} s \omega_{f}^{2}\left[\frac{3}{4} B^{3} \mathrm{e}^{3 \mathrm{i} \omega_{f} T_{0}}+\left(\frac{3}{4} B \bar{B}-1\right) B \mathrm{e}^{\mathrm{i} \omega_{f} T_{0}}\right]+\text { c.c. }
\end{gathered}
$$

where c.c. stands for the complex conjugate of the proceeding terms.

The solvability conditions of Eqs. (3.6) can be obtained by equating the coefficients of secular terms to zero, which reads

$$
\begin{aligned}
& b B \mathrm{e}^{\mathrm{i} \sigma_{1} T_{1}}+\widetilde{d} \Omega_{0}^{2} B \mathrm{e}^{\mathrm{i} \sigma_{1} T_{1}}-\mathrm{i} \zeta \omega_{s} A-a \Omega_{0} \Omega_{1} \bar{A} \mathrm{e}^{\mathrm{i} \sigma T_{1}}-2 \mathrm{i} \omega_{s} D_{1} A=0 \\
& -M \omega_{s}^{2} A \mathrm{e}^{-\mathrm{i} \sigma_{1} T_{1}}-\mathrm{i} s \omega_{f}^{2}\left(\frac{3}{4} B \bar{B}-1\right) B-2 \mathrm{i} \omega_{f} D_{1} B=0
\end{aligned}
$$

The derivatives of amplitudes $A$ and $B$ with respect to $T_{1}$ can be obtained by Eqs. (3.7), that is

$$
\begin{aligned}
& D_{1} A=\frac{1}{2 \omega_{s}}\left[-\mathrm{i} b B \mathrm{e}^{\mathrm{i} \sigma_{1} T_{1}}-\mathrm{i} \widetilde{d} \Omega_{0}^{2} B \mathrm{e}^{\mathrm{i} \sigma_{1} T_{1}}-\zeta \omega_{s} A+\mathrm{i} a \Omega_{0} \Omega_{1} \bar{A} \mathrm{e}^{\mathrm{i} \sigma T_{1}}\right] \\
& D_{1} B=\frac{1}{2 \omega_{f}}\left[\mathrm{i} M \omega_{s}^{2} A \mathrm{e}^{-\mathrm{i} \sigma_{1} T_{1}}-s \omega_{f}^{2}\left(\frac{3}{4} B \bar{B}-1\right) B\right]
\end{aligned}
$$

Assume that the functions $A$ and $B$ are expressed in polar co-ordinates, which reads

$$
A\left(T_{1}\right)=\frac{a_{1}\left(T_{1}\right)}{2} \mathrm{e}^{\mathrm{i} \theta_{1}\left(T_{1}\right)} \quad B\left(T_{1}\right)=\frac{a_{2}\left(T_{1}\right)}{2} \mathrm{e}^{\mathrm{i} \theta_{2}\left(T_{1}\right)}
$$


where $a_{k}, \theta_{k}(k=1,2)$ represent the amplitudes and phase angles of the responses, respectively. The first-order averaged equations can be obtained after separating the real and imaginary parts by substituting (3.9) into Eqs. (3.8), that is

$$
\begin{aligned}
a_{1}^{\prime} & =\frac{1}{2 \omega_{s}}\left[\left(b+\widetilde{d} \Omega_{0}^{2} t\right) a_{2} \sin \varphi-\zeta \omega_{s} a_{1}-a \Omega_{0} \Omega_{1} a_{1} \sin \phi\right] \\
\theta_{1}^{\prime} & =\frac{1}{2 a_{1} \omega_{s}}\left[-\left(b+\widetilde{d} \Omega_{0}^{2}\right) a_{2} \cos \varphi+a \Omega_{0} \Omega_{1} a_{1} \cos \phi\right] \\
a_{2}^{\prime} & =\frac{1}{2 \omega_{f}}\left[M \omega_{s}^{2} a_{1} \sin \varphi-s \omega_{f}^{2}\left(\frac{3}{16} a_{2}^{2}-1\right) a_{2}\right] \\
\theta_{2}^{\prime} & =\frac{1}{2 a_{2} \omega_{f}} M \omega_{s}^{2} a_{1} \cos \varphi
\end{aligned}
$$

where $\left({ }^{\prime}\right)$ denotes the derivatives with respect to $T_{1}$ and $\varphi=\theta_{2}+\sigma_{1} T_{1}-\theta_{1}, \phi=\sigma T_{1}-2 \theta_{1}$.

The derivatives of $\varphi$ and $\phi$ with respect to $T_{1}$ can be derived by eliminating $\theta_{1}$ and $\theta_{2}$ from Eqs. $(3.10)_{2,4}$

$$
\begin{aligned}
\varphi^{\prime} & =\frac{M \omega_{s}^{2} a_{1} \cos \varphi}{2 a_{2} \omega_{f}}+\sigma_{1}+\frac{\left(b+\widetilde{d} \Omega_{0}^{2}\right) a_{2} \cos \varphi-a \Omega_{0} \Omega_{1} a_{1} \cos \phi}{2 a_{1} \omega_{s}} \\
\phi^{\prime} & =\sigma+\frac{\left(b+\widetilde{d} \Omega_{0}^{2}\right) a_{2} \cos \varphi-a \Omega_{0} \Omega_{1} a_{1} \cos \phi}{a_{1} \omega_{s}}
\end{aligned}
$$

The equilibrium solutions of Eqs. (3.10) $)_{1,3}$ and (3.11) correspond to periodic motions of the coupled system. The steady-state solutions for system (2.8) can be obtained when assuming $a_{1}^{\prime}=0, a_{2}^{\prime}=0, \varphi^{\prime}=0, \phi^{\prime}=0$.

\section{The nonlinear response and bifurcation analysis with different system parameters}

The research of Facchinetti et al. (2004), Keber and Wiercigroch (2008), Wang et al. (2016c) showed that during the interaction process of the fluid and structure, the structural motion can affect formation of the fluid as well. Therefore, the effects of the system parameters including the detuning parameter $\sigma$, steady-state rotating speed $\Omega_{0}$ and the amplitude of periodic perturbation $\Omega_{1}$ as well as freestream velocity $V$ on the amplitudes and phase angles of the responses under different coupling parameters $M$ are investigated. The bifurcation curves are computed and stability is determined by examining the eigenvalues of the corresponding characteristic equation to Eqs. $(3.10)_{1,3}$ and (3.11).

Figures 2 and 3 show the varying trends of the amplitudes $a_{1}$ and $a_{2}$ and phase angles $\varphi$ and $\phi(\bmod T)$ with respect to the detuning parameter $\sigma$ for the coupling parameters $M=0.1,0.2,0.3$, respectively. The other parameters are fixed at $A=4.2 \cdot 10^{-4} \mathrm{~m}^{2}$, $\rho=7800 \mathrm{~kg} / \mathrm{m}^{3}, \rho_{f}=1.225 \mathrm{~kg} / \mathrm{m}^{3}, E I=300 \mathrm{Nm}, V=110 \mathrm{~m} / \mathrm{s}, \quad \Omega_{0}=350 \mathrm{rad} / \mathrm{s}$, $\Omega_{1}=0.1 \mathrm{rad} / \mathrm{s}, \bar{D}=0.1 \mathrm{~m}, r=0.3 \mathrm{~m}, \widetilde{c}=6 \mathrm{~N} \mathrm{~s} / \mathrm{m}, C_{L 0}=0.01, \omega_{f}=552.64 \mathrm{rad} / \mathrm{s}, s=0.03$, respectively.

It can be seen from Figs. 2a,b and 3a,b that as the detuning parameter $\sigma$ increases, the trivial solutions of the amplitudes $a_{1}, a_{2}$ and the phase angles $\varphi, \phi$ jump to large two-mode solutions via a saddle-node bifurcation at $S N_{1}$, leading to the occurrence of a stable and an unstable solution. Similarly, as the dutuning parameter $\sigma$ decreases, the trivial solutions of the amplitudes $a_{1}$ and $a_{2}$ and the phase angles $\varphi, \phi$ become other two-mode solutions via a saddle-node bifurcation at $S N_{2}$, resulting in a stable and an unstable solution, respectively. 
(a)

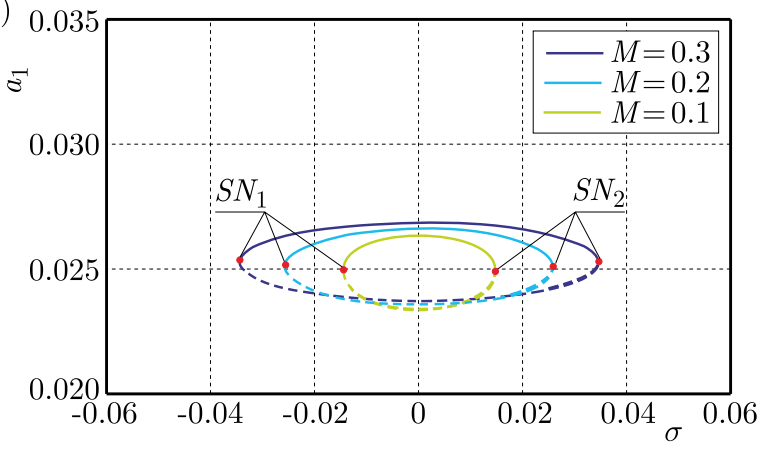

(b)

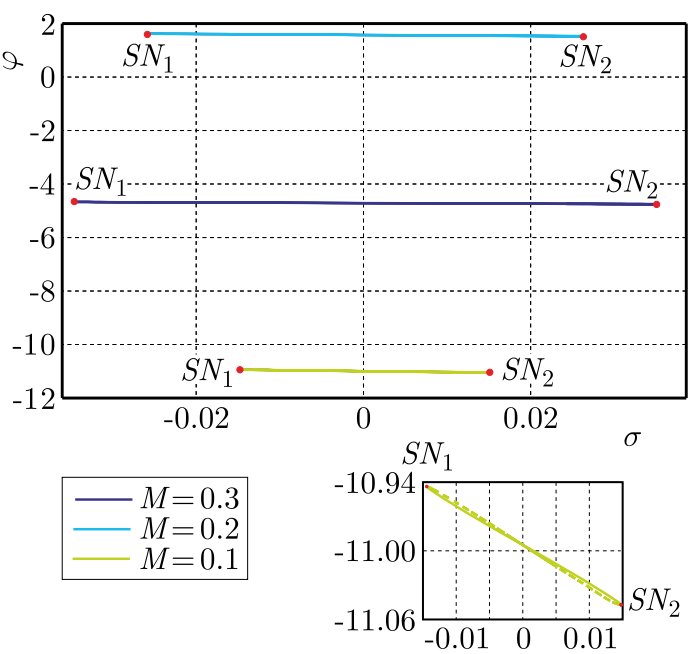

Fig. 2. Frequency-response curves of the amplitude $a_{1}$ and the phase angle $\varphi$ of the structure with respect to the detuning parameter $\sigma$

(a)

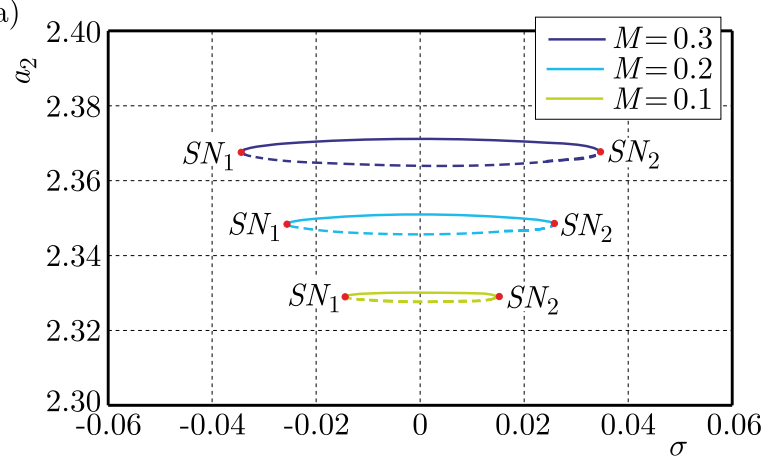

(b)

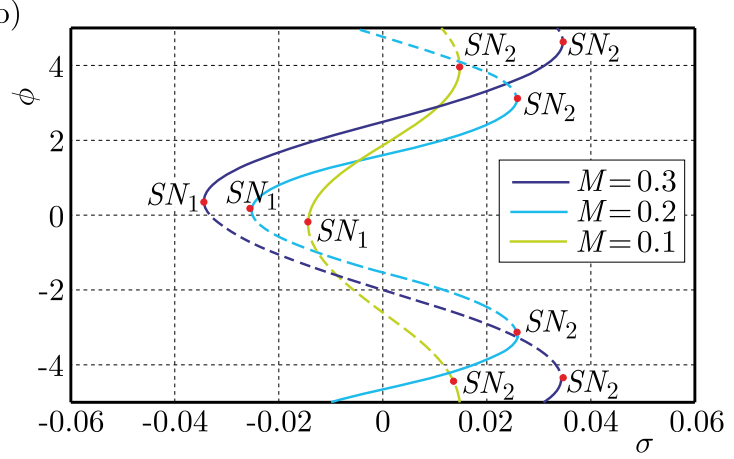

Fig. 3. Bifurcation curves of the amplitude $a_{2}$ and the phase angle $\phi$ of the fluid with respect to the detuning parameter $\sigma$

Figures $2 \mathrm{a}$ and $3 \mathrm{a}$ show that the amplitudes $a_{1}$ and $a_{2}$ have the same varying trends with respect to the varying detuning parameter. The phase angles $\varphi$ and $\phi$ have the opposite varying trends with respect to the varying detuning parameter, which means that there is transformation between the two vibration modes. In addition, Figs. 2 and 3 show that the absolute value of $\sigma$ for the critical bifurcation increases and the amplitudes as well as the period of the steady-state solutions can be increased as the coupling parameter $M$ increases.

Figures 4 and 5 show the varying trends of the responses with respect to the steady-state rotating speed $\Omega_{0}$ for the coupling parameters $M=0.1,0.2,0.3$, respectively. The other parameters are fixed at $A=4.2 \cdot 10^{-4} \mathrm{~m}^{2}, \rho=7800 \mathrm{~kg} / \mathrm{m}^{3}, \rho_{f}=1.225 \mathrm{~kg} / \mathrm{m}^{3}, E I=300 \mathrm{~N} \mathrm{~m}, \mathrm{~V}=110 \mathrm{~m} / \mathrm{s}$, $\Omega_{1}=0.1 \mathrm{rad} / \mathrm{s}, \bar{D}=0.1 \mathrm{~m}, r=0.3 \mathrm{~m}, \widetilde{c}=6 \mathrm{~N} \mathrm{~s} / \mathrm{m}, C_{L 0}=0.01, \omega_{f}=552.64 \mathrm{rad} / \mathrm{s}, s=0.03$, $\sigma=0.01$, respectively.

It can be seem from Figs. 4 and 5 that the trivial solution jumps to large solutions via a saddle-node bifurcation, resulting in the occurrence of a two-mode solution consisting of a stable solution and an unstable one. Figure 4a shows that the steady-state solutions of the amplitude $a_{1}$ decrease as the steady-state rotating speed $\Omega_{0}$ increases, which indicates that the increasing of the steady-state rotating speed can suppress the large-amplitude vibrations of the structure. Figure 5a shows that the steady-state solutions of the amplitude $a_{2}$ increase as the steady-state rotating speed $\Omega_{0}$ increases, which displays an inverse varying trend of the responses comparing with those for the amplitude $a_{1}$. By comparison, the stable and unstable solutions for the phase angle $\varphi$ as shown in Fig. 4b increase simultaneously as the steady-state rotating 

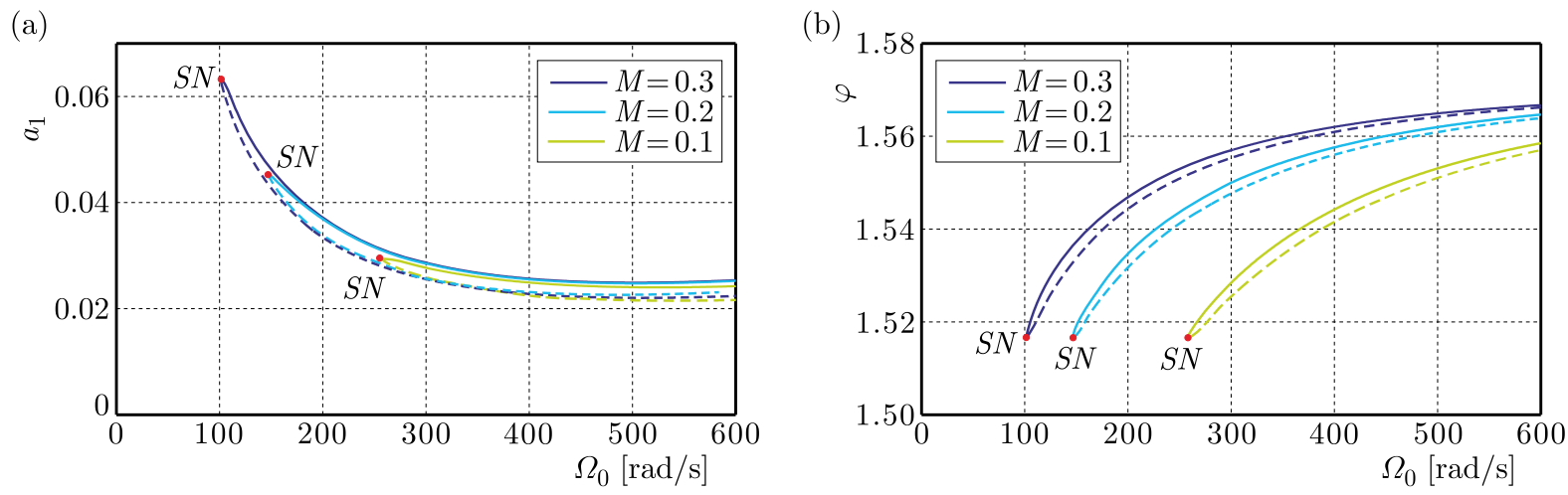

Fig. 4. Bifurcation curves of the amplitude $a_{1}$ and the phase angle $\varphi$ of the structure with respect to the steady-state rotating speed $\Omega_{0}$

(a)

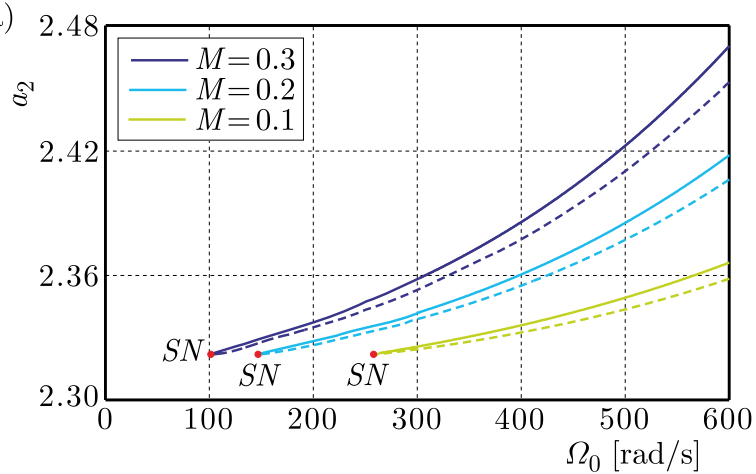

(b)

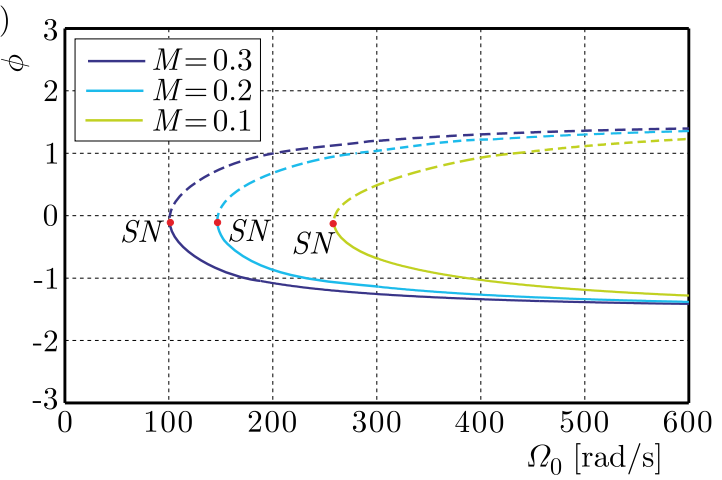

Fig. 5. Bifurcation curves of the amplitude $a_{2}$ and the phase angle $\phi$ of the fluid with respect to the steady-state rotating speed $\Omega_{0}$

speed $\Omega_{0}$ increases while the stable solutions of the phase angle $\phi$ decrease and the unstable one increases when the steady-state rotating speed $\Omega_{0}$ increases, as shown in Fig. 5b. Moreover, the critical steady-state rotating speed can decrease for the saddle-node bifurcation as the coupling parameter $M$ increases, that is: $\Omega_{0}=257.9793 \mathrm{rad} / \mathrm{s}$ for $M=0.1, \Omega_{0}=146.8258 \mathrm{rad} / \mathrm{s}$ for $M=0.2, \Omega_{0}=101.3993 \mathrm{rad} / \mathrm{s}$ for $M=0.3$, respectively. In addition, an increase in the coupling parameter $M$ can increase the amplitudes $a_{1}$ and $a_{2}$ of the responses. It can be illustrated from system (2.8) that the increasing of the coupling parameter $M$ can excite large vibrations of the fluid, which can in turn promote the oscillations of the structure.

Figures 6 and 7 show the bifurcation characteristics of the system responses with respect to the amplitude $\Omega_{1}$ of the periodic perturbation for different coupling parameters $M$. The other parameters are fixed at $A=4.2 \cdot 10^{-4} \mathrm{~m}^{2}, \rho=7800 \mathrm{~kg} / \mathrm{m}^{3}, \rho_{f}=1.225 \mathrm{~kg} / \mathrm{m}^{3}, E I=300 \mathrm{~N} \mathrm{~m}$, $V=110 \mathrm{~m} / \mathrm{s}, \Omega_{0}=350 \mathrm{rad} / \mathrm{s}, \bar{D}=0.1 \mathrm{~m}, r=0.3 \mathrm{~m}, \widetilde{c}=6 \mathrm{~N} \mathrm{~s} / \mathrm{m}, C_{L 0}=0.01, \omega_{f}=552.64 \mathrm{rad} / \mathrm{s}$, $s=0.03, \sigma=0.01$, respectively.

It can be seen from Figs. 6 and 7 that the trivial solution jumps to the large solution via a saddle-node bifurcation $(S N)$, leading to the occurrence of a two-mode solution including a stable solution and an unstable one as the parameter $\Omega_{1}$ increases. Figures $6 \mathrm{a}$ and $7 \mathrm{a}$ display that the varying trends of the amplitudes $a_{1}$ and $a_{2}$ with respect to the parameter $\Omega_{1}$ are the same to each other, that is, the stable solutions of the amplitudes $a_{1}$ and $a_{2}$ increase as $\Omega_{1}$ increases while the unstable solutions decrease as $\Omega_{1}$ increases. In comparison, the stable and unstable solutions of the phase angles $\varphi$ and $\phi$ have the opposite varying trend. In addition, the increasing of the coupling parameter $M$ can make the critical bifurcation value of the parameter $\Omega_{1}$ smaller, that is, $\Omega_{1}=0.0674 \mathrm{rad} / \mathrm{s}$ for $M=0.1, \Omega_{1}=0.0383 \mathrm{rad} / \mathrm{s}$ for $M=0.2$ and $\Omega_{1}=0.0286 \mathrm{rad} / \mathrm{s}$ for $M=0.3$, respectively. 
(a)

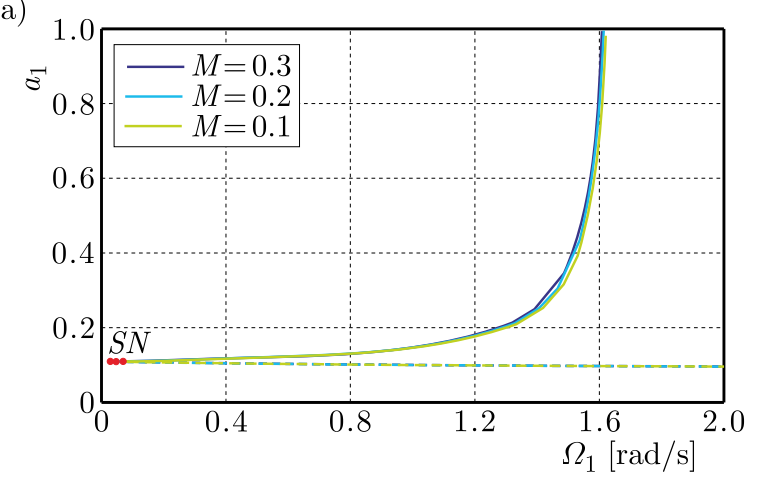

(b)

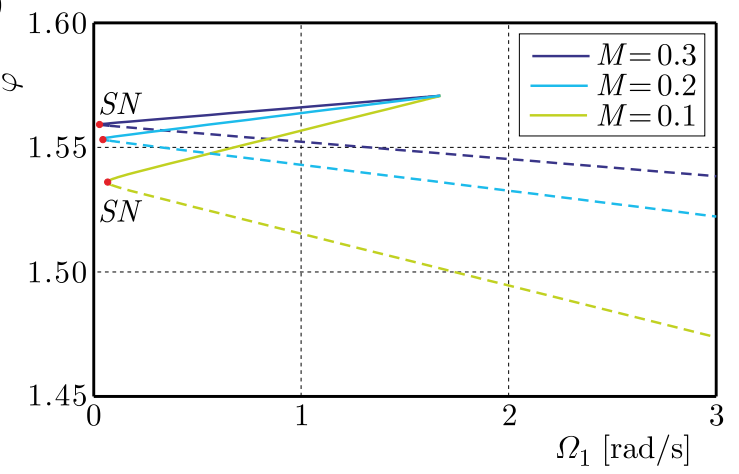

Fig. 6. Bifurcation curves of the amplitude $a_{1}$ and the phase angle $\varphi$ of the structure with respect to the amplitude $\Omega_{1}$ of the periodic perturbation

(a)

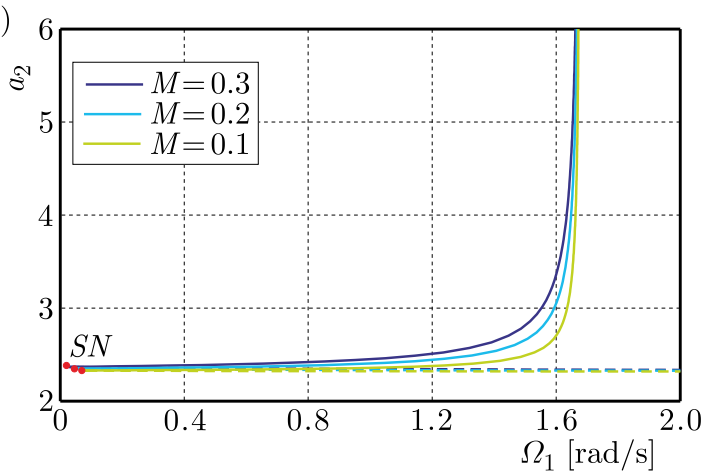

(b)

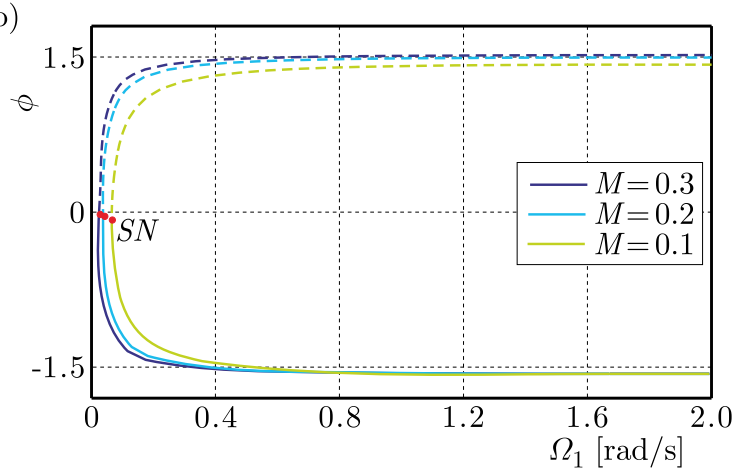

Fig. 7. Bifurcation curves of the amplitude $a_{2}$ and the phase angle $\phi$ of the fluid with respect to the amplitude $\Omega_{1}$ of the periodic perturbation

Figures 8 and 9 display the varying trends of the responses with respect to the freestream velocity $V$ under different values of the coupling parameter $M$. The other parameters are fixed at $A=4.2 \cdot 10^{-4} \mathrm{~m}^{2}, \rho=7800 \mathrm{~kg} / \mathrm{m}^{3}, \rho_{f}=1.225 \mathrm{~kg} / \mathrm{m}^{3}, E I=300 \mathrm{~N} \mathrm{~m}, \Omega_{0}=350 \mathrm{rad} / \mathrm{s}$, $\Omega_{1}=0.1 \mathrm{rad} / \mathrm{s}, \bar{D}=0.1 \mathrm{~m}, r=0.3 \mathrm{~m}, \widetilde{c}=6 \mathrm{~N} \mathrm{~s} / \mathrm{m}, C_{L 0}=0.01, s=0.03, \sigma=0.01$, respectively.

(a)

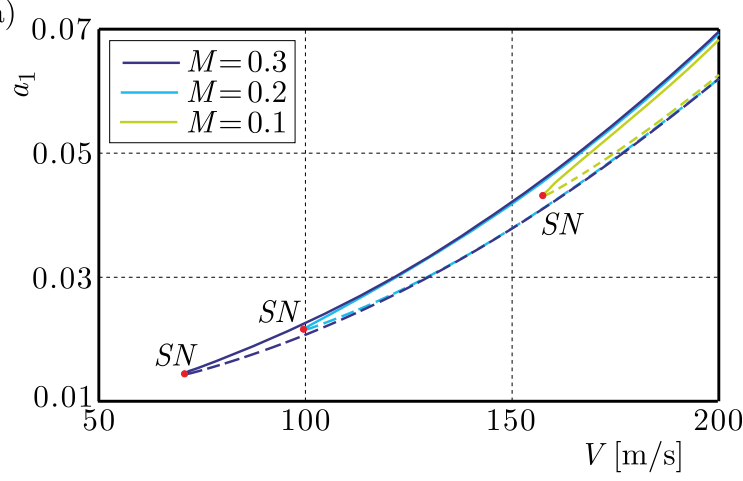

(b)

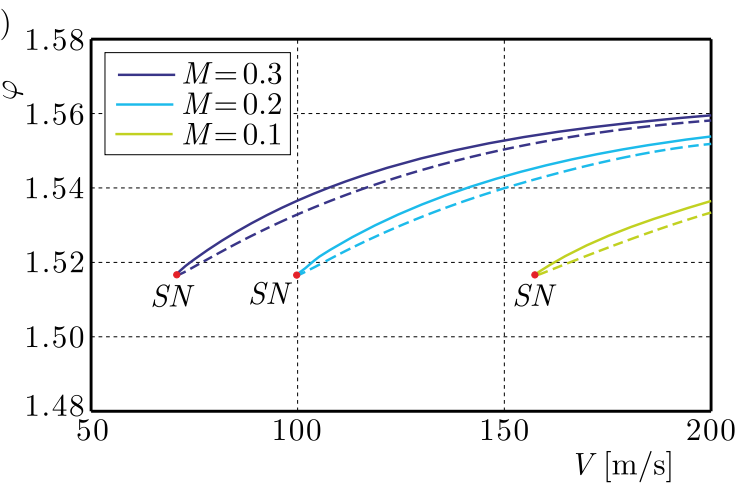

Fig. 8. Bifurcation curves of the amplitude $a_{1}$ and the phase angle $\varphi$ of the structure with respect to the freestream velocity $V$

It can be seen from Figs. 8 and 9 that the trivial solution jumps to a large solution via a saddle-node bifurcation as the freestream velocity $V$ increases, resulting in the occurrence of a two-mode solution consisting of a stable solution and an unstable one. The stable and unstable solutions of the amplitudes $a_{1}$ and $a_{2}$ increase when the freestream velocity $V$ increases, as shown in Figs. 8a and 9a which present the similar varying trends for the solutions of the amplitudes 
(a)

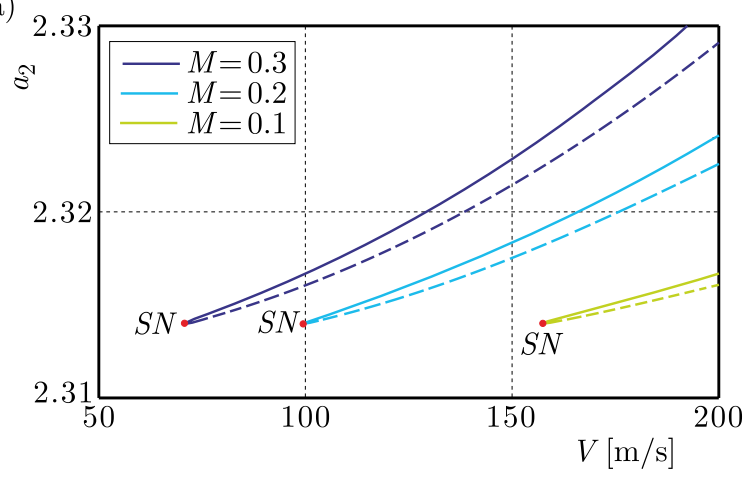

(b)

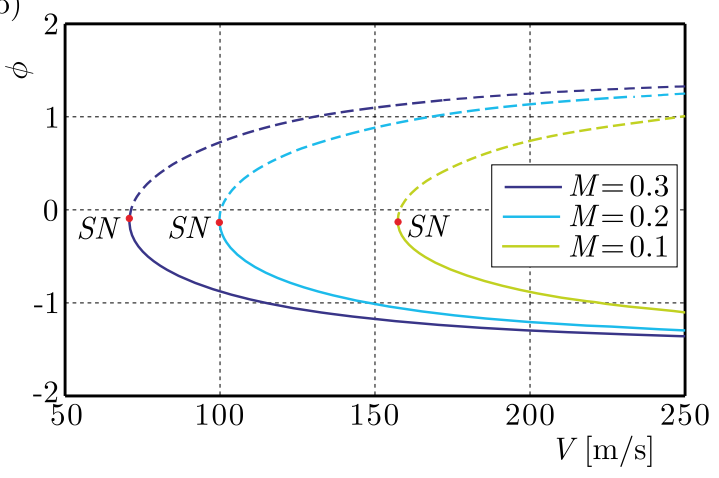

Fig. 9. Bifurcation curves of the amplitude $a_{2}$ and the phase angle $\phi$ of the fluid with respect to the freestream velocity $V$

$a_{1}$ and $a_{2}$. Figures $8 \mathrm{~b}$ and $9 \mathrm{~b}$ indicate the opposite varying trends for the stable solutions of the phase angles $\varphi$ and $\phi$, namely, the solution of the phase angle $\varphi$ increases while the solution of the phase angle $\phi$ decreases as the freestream velocity $V$ increases. Similarly, the increasing of the coupling parameter $M$ can decrease the critical freestream velocity $V$ for the saddle-node bifurcation of the responses.

\section{Conclusions}

The dynamic responses and bifurcation characteristics of turbine blades under variable rotating speed have been investigated, where the rotating blade was modeled as a cantilever beam and the effect of the oncoming vortices was represented as the van der Pol oscillation. And the acceleration coupling was considered to simulate the influence of the vibration of the beam on the van der Pol oscillation. The first-order mode vibrations of the coupled system were obtained by the Galerkin discretization. The 1:2 subharmonic resonance and the 1:1 internal resonance for the coupled system were studied by using the multiple scale method. The averaged equations were derived and the bifurcation curves were computed. Effects of the system parameters including the dutuning parameter $\sigma$, the steady-state rotating speed $\Omega_{0}$, the amplitude $\Omega_{1}$ of the periodic perturbation as well as the freestream velocity $V$ on the responses were investigated.

The phenomenon of saddle-node bifurcation was found to occur under certain parameter conditions. The bifurcation analysis indicates that the increasing of the coupling parameter $M$ can delay the saddle-node bifurcation of the responses with respect to the detuning parameter while the increasing of the coupling parameter $M$ can make the saddle-node bifurcation occur earlier for the responses with respect to the steady-state rotating speed $\Omega_{0}$, the amplitude $\Omega_{1}$ of the periodic perturbation as well as the freestream velocity $V$. Moreover, the increasing of the steady-state rotating speed can suppress large vibration of the structure. The amplitudes of vibrations of the structure and fluid can be increased as the freestream velocity $V$ increases. The opposite varying trends for the amplitudes and phase angles with respect to the system parameters indicate the energy transfer between the vibrations of the fluid and the structure. The results can help one to understand the interaction of the fluid and the structure.

\section{Acknowledgement}

The authors acknowledge the financial support from the National Natural Science Foundation of China (No. 11702111, 11572205, 11732014), the Natural Science Foundation of Shandong Province (No. ZR2017QA005, ZR2017BA031) and the University of Jinan (No. 160100210). 


\section{References}

1. Barron M.A., Sen M., 2009, Synchronization of coupled self-excited elastic beams, Journal of Sound and Vibration, 324, 209-220

2. Barron M.A., 2010, Vibration analysis of a self-excited elastic beam, Journal of Applied Research and Technology, 8, 2, 227-239

3. Clough R.W., Penzien J., 2003, Dynamics of structures, Computers and Structures, Inc., 3rd edition

4. Domagalski Ł., JęDrysiak J., 2016, Nonlinear vibrations of periodic beams, Journal of Theoretical and Applied Mechanics, 54, 4, 1095-1108

5. Facchinetti M.L., De Langree E., Biolley F., 2004, Coupling of structure and wake oscillators in vortex-induced vibrations, Journal of Fluids and Structures, 19, 123-140

6. Gabbai R., Benaroya H., 2005, An overview of modeling and experiments of vortex-induced vibration of circular cylinders, Journal of Sound and Vibration, 282, 575-616

7. Georgiades F., Latalski J., Warmiński J., 2014, Equations of motion of rotating composite beam with a nonconstant rotation speed and an arbitrary preset angle, Meccanica, 49, 1833-1858

8. Gostelow J.P., Platzer M.F., Carscallen W.E., 2006, On vortex formation in the wake flows of transonic turbine blades and oscillating airfoils, Journal of Turbomachinery-ASME, 128, 528-535

9. Hartlen R., Currie I., 1970, Lift-oscillator model of vortex induced vibration, Journal of Engineering Mechanics-ASCE, 96, 577-591

10. Hao Z.F., CaO Q.J., 2015, The isolation characteristics of an archetypal dynamical model with stable-quasi-zero-stiffness, Journal of Sound and Vibration, 340, 61-79

11. HaO Z.F., CaO Q.J., Wiercigroch M., 2016, Two-sided damping constraint control for high-performance vibration isolation and end-stop impact protection, Nonlinear Dynamics, 86, 2129-2144

12. Hemon P., 1999, An improvement of the time delayed quasi-steady model for the oscillations of circular cylinders in cross-flow, Journal of Fluids and Structures, 13, 291-307

13. Kammer D.C., Schlack A.L., 1987, Effects of nonconstant spin rate on the vibration of a rotating beam, Journal of Applied Mechanics, 54, 2, 305-310

14. Keber M., Wiercigroch M., 2008, A reduced order model for vortex-induced vibration of a vertical offshore riser in lock-in, IUTAM Symposium on Fluid-Structure Interaction in Ocean Engineering, Iutam Bookseries, 8, 155-166

15. Kim H., Chung J., 2016, Nonlinear modeling for dynamic analysis of a rotating cantilever beam, Nonlinear Dynamics, 86, 1981-2002

16. Lee Y., Vakakis A., Bergman L., McFarland M., 2006, Suppression of limit cycle oscillations in the van der Pol oscillator by means of passive nonlinear energy sinks, Structural Control and Health Monitoring, 13, 41-75

17. NAYFeh A.H., Mook D.T., 1979, Nonlinear Oscillations, New York, 331-338

18. Shahlaei-Far S., Nabarrete A., Balthazar J.M., 2016, Homotopy analysis of a forced nonlinear beam model with quadratic and cubic nonlinearities, Journal of Theoretical and Applied Mechanics, 54, 4, 1219-1230

19. Staino A., Basu B., 2013, Dynamics and control of vibrations in wind turbines with variable rotor speed, Engineering Structures, 56, 58-67

20. Wang D., Chen Y.S., Wiercigroch M., CaO Q.J., 2016a, A three-degree-of-freedom model for vortex-induced vibrations of turbine blades, Meccanica, 51, 2607-2628 
21. Wang D., Chen Y.S., Hao Z.F., CaO Q.J., 2016b, Bifurcation analysis for vibrations of a turbine blade excited by air flows, Science China Technological Sciences, 59, 8, 1217-1231

22. Wang D., Chen Y.S., Wiercigroch M., CaO Q.J., 2016c, Bifurcation and dynamic response analysis of rotating blade excited by upstream vortices, Applied Mathematics and Mechanics, 37, $9,1251-1274$

23. WARmiński J., LAtalski J., 2016, Saturation control for a rotating thin-walled composite beam structure, Procedia Engineering, 144, 713-720

24. Wei K., Meng G., Zhou S., Liu J., 2006, Vibration control of variable speed/acceleration rotating beams using smart materials, Journal of Sound and Vibration, 298, 4/5, 1150-1158

25. Yang J.B., Jiang L.J., Chen D.C., 2004, Dynamic modelling and control of a rotating Euler-Bernoulli beam, Journal of Sound and Vibration, 274, 863-875

26. Yao M.H., Chen Y.P., Zhang W., 2012, Nonlinear vibrations of blade with varying rotating speed, Nonlinear Dynamics, 68, 487-504

27. Yao M.H., Zhang W., Chen Y.P., 2014, Analysis on nonlinear oscillations and resonant responses of a compressor blade, Acta Mechanica, 225, 3483-3510

28. Younesian D., Esmailzadeh E., 2010, Non-linear vibration of variable speed rotating viscoelastic beams, Nonlinear Dynamics, 60, 193-205

29. Zhu K., Chung J., 2016, Dynamic modeling and analysis of a spinning Rayleigh beam under deployment, International Journal of Mechanical Sciences, 115-116, 392-405

Manuscript received January 10, 2017; accepted for print July 15, 2017 